\title{
Potential anti-tumor immune response mechanisms of SARS-CoV-2 in lymphomas: can the devil be converted into a boon?
}

\section{Debmalya Barh ( $\sim$ dr.barh@gmail.com )}

Institute of Integrative Omics and Applied Biotechnology (IIOAB), Nonakuri, Purba Medinipur, West Bengal-721172, India; and Department of Genetics, Ecology and Evolution, Institute of Biological Sciences, Federal University of Minas Gerais, Belo Horizonte 31270-901, Brazil https://orcid.org/00000002-2557-7768

\section{Sandeep Tiwari}

Federal University of Minas Gerais, Belo Horizonte 31270-901, Brazil

\section{Lucas Gabriel Rodrigues Gomes}

Federal University of Minas Gerais, Belo Horizonte 31270-901, Brazil

\section{Marianna E. Weener}

Clinical Research Center, Oftalmic, CRO, 119334 Bardina Str. 22/4, Moscow, Russia

\section{Khalid J. Alzahrani}

Department of Clinical Laboratories Sciences, College of Applied Medical Sciences, Taif University, Taif 21944, Saudi Arabia

\section{Khalaf F. Alsharif}

Department of Clinical Laboratories Sciences, College of Applied Medical Sciences, Taif University, Taif 21944, Saudi Arabia

\section{Alaa A. A. Aljabali}

Department of Pharmaceutics and Pharmaceutical Technology, Faculty of Pharmacy, Yarmouk University, Irbid 21163, Jordan

\section{Murtaza M. Tambuwala}

School of Pharmacy and Pharmaceutical Science, Ulster University, Coleraine BT52 1SA, UK

\section{Kenneth Lundstrom}

PanTherapeutics, CH 1095 Lutry, Switzerland

\section{Sk. Sarif Hassan}

Department of Mathematics, Pingla Thana Mahavidyalaya, Maligram, 721140, India

\section{Ángel Serrano-Aroca}

Centro de Investigación Traslacional San Alberto Magno, Universidad Católica de Valencia San Vicente Mártir, 46001 Valencia, Spain

\section{Kazuo Takayama}

Center for iPS Cell Research and Application (CiRA), Kyoto University, Kyoto 606-8507, Japan 


\section{Preetam Ghosh}

Department of Computer Science, Virginia Commonwealth University, Richmond, Virginia, USA

\section{Elrashdy M. Redwan}

King Abdulazizi University, Faculty of Science, Department of Biological Science, Saudi Arabia

\section{Bruno Silva Andrade}

Laboratório de Bioinformática e Química Computacional, Departamento de Ciências Biológicas, Universidade Estadual do Sudoeste da Bahia (UESB), Jequié 45206-190, Brazil.

\section{Siomar de Castro Soares}

Department of Immunology, Microbiology and Parasitology, Institute of Biological and Natural Sciences, Federal University of Triângulo Mineiro (UFTM), Uberaba 38025-180, MG, Brazil

\section{Vasco Azevedo}

Department of Genetics, Ecology and Evolution, Institute of Biological Sciences, Federal University of Minas Gerais, Belo Horizonte 31270-901, Brazil

\section{Vladimir N. Uversky}

Department of Molecular Medicine and USF Health Byrd Alzheimer's Institute, Morsani College of Medicine, University of South Florida, Tampa, FL 33612, USA

\section{Research Article}

Keywords: Lymphoma, SARS-CoV-2, M protein, Orf3a, anti-tumor immunotherapy, gamma-tubulin ring complex, monoclonal antibody

Posted Date: August 17th, 2021

DOl: https://doi.org/10.21203/rs.3.rs-811181/v1

License: (c) (1) This work is licensed under a Creative Commons Attribution 4.0 International License. Read Full License 


\title{
Potential anti-tumor immune response mechanisms of SARS- CoV-2 in lymphomas: can the devil be converted into a boon?
}

\author{
Debmalya Barh ${ }^{1,2 \dagger^{\star}}$, Sandeep Tiwari ${ }^{2 \dagger}$, Lucas Gabriel Rodrigues Gomes ${ }^{2}$, Marianna E. Weener ${ }^{3}$, \\ Khalid J. Alzahrani ${ }^{4}$, Khalaf F. Alsharif ${ }^{4}$, Alaa A. A. Aljabali ${ }^{5}$, Murtaza M. Tambuwala ${ }^{6}$, Kenneth \\ Lundstrom $^{7}$, Sk. Sarif Hassan ${ }^{8}$, Ángel Serrano-Aroca ${ }^{9}$, Kazuo Takayama ${ }^{10}$, Preetam Ghosh ${ }^{11}$, \\ Elrashdy M. Redwan ${ }^{12}$, Bruno Silva Andrade ${ }^{13}$, Siomar de Castro Soares ${ }^{14}$, Vasco Azevedo, \\ Vladimir N. Uversky ${ }^{15}$
}

${ }^{1}$ Centre for Genomics and Applied Gene Technology, Institute of Integrative Omics and Applied Biotechnology (IIOAB), Nonakuri, Purba Medinipur, West Bengal-721172, India

${ }^{2}$ Department of Genetics, Ecology and Evolution, Institute of Biological Sciences, Federal University of Minas Gerais, Belo Horizonte 31270-901, Brazil

${ }^{3}$ Clinical Research Center, Oftalmic, CRO, 119334 Bardina Str. 22/4, Moscow, Russia

${ }^{4}$ Department of Clinical Laboratories Sciences, College of Applied Medical Sciences, Taif University, Taif 21944, Saudi Arabia

${ }^{5}$ Department of Pharmaceutics and Pharmaceutical Technology, Faculty of Pharmacy, Yarmouk University, Irbid 21163, Jordan

${ }^{6}$ School of Pharmacy and Pharmaceutical Science, Ulster University, Coleraine BT52 1SA, UK

${ }^{7}$ PanTherapeutics, CH 1095 Lutry, Switzerland

${ }^{8}$ Department of Mathematics, Pingla Thana Mahavidyalaya, Maligram, 721140 , India

9Biomaterials and Bioengineering Lab, Centro de Investigación Traslacional San Alberto Magno, Universidad Católica de Valencia San Vicente Mártir, 46001 Valencia, Spain

${ }^{10}$ Center for iPS Cell Research and Application (CiRA), Kyoto University, Kyoto 606-8507, Japan

${ }^{11}$ Department of Computer Science, Virginia Commonwealth University, Richmond, Virginia, USA

${ }^{12}$ King Abdulazizi University, Faculty of Science, Department of Biological Science, Saudi Arabia

${ }^{13}$ Laboratório de Bioinformática e Química Computacional, Departamento de Ciências Biológicas, Universidade Estadual do Sudoeste da Bahia (UESB), Jequié 45206-190, Brazil.

${ }^{14}$ Department of Immunology, Microbiology and Parasitology, Institute of Biological and Natural Sciences, Federal University of Triângulo Mineiro (UFTM), Uberaba 38025-180, MG, Brazil

${ }^{15}$ Department of Molecular Medicine and USF Health Byrd Alzheimer's Institute, Morsani College of Medicine, University of South Florida, Tampa, FL 33612, USA

${ }^{\dagger}$ These authors contributed equally.

*Corresponding author Email-dr.barh@gmail.com 


\section{ABSTRACT}

Recently, two cases of complete remission of classical Hodgkin lymphoma (cHL) and follicular lymphoma (FL) after SARS-CoV-2 infection were reported. However, the precise molecular mechanism of this rare event is yet to be understood. Here, we hypothesize a potential anti-tumor immune response of SARS-CoV-2 and based on computational approach show that (i) SARS-CoV-2 Spike-RBD may bind to extracellular domains of CD15, CD27, CD45, and CD152 receptors of $\mathrm{CHL}$ or $\mathrm{FL}$, (ii) upon internalization, SARS-CoV-2 membrane (M) protein and Orf3a may bind to gamma-tubulin complex component 3 (GCP3) at its tubulin gamma-1 chain (TUBG1) binding site, (iii) M protein may also interact with TUBG1 blocking its binding to GCP3, (iv) both M and Orf3a may render the GCP2-GCP3 lateral binding where $M$ possibly interacts with GCP2 at its GCP3 binding site and Orf3a to GCP3 at its GCP2 interacting residues, $(v)$ interactions of $M$ and Orf3a with these gamma-tubulin ring complex components potentially block the initial process of microtubule nucleation, leading to cell cycle arrest and apoptosis, (vi) SpikeRBD may also interact with and block PD-1 signaling similar to pembrolizumab and nivolumab like monoclonal antibodies and may induce B-cell apoptosis and remission, (vii) finally, the TRADD interacting PVQLSY motif of Epstein-Barr virus LMP-1, that is responsible for NF-kB mediated oncogenesis, potentially interacts with SARS-CoV-2 Mpro, nsp7, nsp10, and Spike proteins and may regulate the LMP1 mediated cell proliferation. Taken together, our results suggest a possible therapeutic potential of SARS-CoV-2 in proliferative disorders.

Keywords: Lymphoma, SARS-CoV-2, M protein, Orf3a, anti-tumor immunotherapy, gamma-tubulin ring complex, monoclonal antibody 


\section{INTRODUCTION}

In most cases, cancer is either reported to be a comorbid condition or associated with COVID-19 disease severity from SARS-CoV-2 infection (1). However, it was recently reported that a patient with classical Hodgkin Lymphoma (cHL) showed disease remission upon infection with SARS-CoV-2 (2). A similar observation has been reported in a case of follicular lymphoma (FL) (3). The molecular profiles and the infecting SARS-CoV-2 strains of these patients are unknown, and the authors suggested the complete remission of the diseases is due to an unknown anti-tumor immune response exerted by SARSCoV-2 $(1,2)$. These findings prompted us to elaborate on the molecular mechanisms behind the SARSCoV-2 induced remission of $\mathrm{cHL}$ and $\mathrm{FL}$.

To place the anti-tumor effect of the SARS-CoV-2 in the right context, it is appropriate to mention that several other single-stranded RNA viruses have demonstrated oncolytic effect leading to efficient killing of tumor cells while causing no damage to normal tissue (4). For this reason, alphaviruses, measles viruses, rhabdoviruses, and Newcastle disease viruses have been used in preclinical animal models and in clinical trials showing encouraging results for cancer therapy (5). However, the oncolytic effects of viruses vary. For instance, significantly lower Zing-finger antiviral protein (ZAP) expression in tumors prevented the viral RNA degradation and translational inhibition of the oncolytic M1 alphavirus commonly occurring in normal tissue (6). In the case of measles viruses, it has been demonstrated that CD46 mediates virus attachment, entry, and virus-induced cell-to-cell fusion for the MV-Edmonston strain (7). Typically, the high density of CD46 on tumor cells contributes to extensive cell fusion and enhancement of viral gene expression and provides a compelling explanation for the oncolytic specificity of the MV-Edmonston strain and its favorable use for CD46-targeted cancer therapy. Related to rhabdoviruses, genome-wide RNAi screening identified the endoplasmic reticulum (ER) stress response pathways as important modulators for sensitization to caspase-2-dependent apoptosis and cell death (8). The tumor cell killing mechanisms of Newcastle disease virus are based directly on the formation of multinucleated syncytia, activation of the extrinsic apoptotic pathway, activation of ER stress pathways and involvement of MAPK pathways (9). Indirectly, Newcastle disease virus can induce secretion of proinflammatory cytokines and chemokines, enhanced adhesion of leukocytes, upregulation of $\mathrm{MHC}$ and cell adhesion molecules to activate tumor-specific lymphocytes.

The $\mathrm{CHL}$ neoplastic cell population is called Hodgkin or Reed-Sternberg (HRS) cells that exclusively express CD30 and CD15, a cluster of differentiation (CD) markers. Additionally, CD40, CD80, CD86, and CD95 are expressed in most CHL cases, and CD20, CD45, and CD3 are rarely expressed by the HRS $(10,11)$. However, some reports suggest that CD20 is expressed at very low intensity in 5 to $80 \%$ of cases of $\mathrm{cHL}$ (12). Other expressed proteins by the $\mathrm{cHL}(5-100 \%$ cases) are CD19, CD79a, CD138, paired box protein Pax-5 (PAX5, also known as B-cell-specific transcription factor, BSAP), and interferon regulatory factor (4IRF4, also known as multiple myeloma oncogene 1, MUM1) (12). Gammaglutamyltransferase 1 (GGT1) could also be a potential marker for $\mathrm{cHL}$ (13), and cytotoxic T-lymphocyte associated protein 4 (CTLA-4) is expressed in programmed cell death-1 (PD-1) negative CHL (14). On the other hand, CD19 is exclusively expressed, and CD20 is also found in some FL cases (15).

$\mathrm{cHL}$ cells exclusively express CD30, and brentuximab vedotin (SGN-35 or Adcetris) is an FDAapproved CD30 targeted drug for the treatment of $\mathrm{cHL}(16)$. SGN-35 is an anti-CD30 monoclonal antibody $(\mathrm{mAb})$ attached to an anti-microtubule compound monomethyl auristatin $E$ (MMAE). Upon binding to CD30, SGN-35 is internalized into CHL cells and releases MMAE that binds to tubulin and thus leads to cell cycle arrest and apoptosis (16). However, $20 \%$ to $30 \%$ cases of $\mathrm{cHL}$ were found to relapse (17). Anti-CD20 mAbs such as rituximab is used to treat FL. Anti-CD19 and anti-CD47 mAbs may also be attractive targets for immunotherapy of FL (18).

Genomic amplification 9p24.1 in cHL has been associated with overexpression of programmed cell death 1 ligand 1 (PD-L1) and activation of Janus kinase 1 and signal transducer and activator of transcription (JAK/STAT) signaling for disease relapse. Therefore, blocking PD-L1 and PD-L2 receptor PD-1 could be a better treatment option. More recently, T-cell checkpoint inhibitory mAbs such as 
nivolumab and pembrolizumab against PD-1 are found to increase the overall survival in $\mathrm{CHL}(19,20)$. Both pembrolizumab and nivolumab bind to the PD-L1 binding site of PD-1 and block the access of PDL1 and PD-L2 and thus prevent relapse of CHL (19). FL can also express PD-1 and therefore, PD-1 immune checkpoint blockers such as pembrolizumab can also be promising targeted therapeutics in FL (18).

It is well established that Epstein-Barr virus (EBV) infection is associated with increased risk, pathogenesis, and immuno-compromission in $\mathrm{CHL}$ in the elderly $(21,22)$. EBV-positive $\mathrm{FL}$ is not rare, and EBV may play a specific role in disease progression and/or lymphomagenesis (23). The latent oncogenic membrane protein 1 (LMP-1) of EBV activates NF-KB, JAK/STAT, and PI3K/AKT pathways, leading to apoptosis-prone germinal center (GC) B-cells and preventing plasma cell differentiation (21). The cytoplasmic signaling domain of LMP-1 recruits tumor necrosis factor receptor-associated factors (TRAFs) and tumor necrosis factor receptor type 1-associated DEATH domain protein (TRADD) to activate NF-kB signaling-mediated B-cell proliferation (24).

The patient was EBV positive in the $\mathrm{CHL}$ case report, although no genetic or molecular profiles were provided (2). Neither have any information in the case of FL been reported (3). Therefore, it is difficult to evaluate the underlying molecular mechanism of the disease remission due to SARS-CoV-2 infection. Hence, we considered all possible genetic makeup of these patients and hypothesized that (i) SARS-CoV-2 brings remission in cHL/FL cells using a similar mechanism as brentuximab vedotin (SGN35 or Adcetris) does. In this process, we presumed that: (a) the SARS-CoV-2 may bind to the extracellular domain of any $\mathrm{CHL}$ specific cell surface CD marker using its Spike-RBD to attach and enter into the lymphoma cells. Upon internalization, some protein of SARS-CoV-2 may interact with cell division-related proteins to bring apoptosis or cell cycle arrest or stop cell division. Alternatively, (b) any protein of SARS-CoV-2 may bind to PD-1 similar to the targeted mAb drugs pembrolizumab and nivolumab and induce remission. The third possibility is that (c) as the patient was positive for EBV, any SARS-CoV-2 protein may interact with the LMP-1 of EBV upon internalization of SARS-CoV-2 into the cell to inhibit the interaction of LMP-1- TRAFs and/or TRADD from abolishing the LMP-1 mediated cell proliferation or cell de-differentiation.

\section{METHODS}

\section{Used protein structures and 3D modeling}

Since the SARS-CoV-2 strains of the infected lymphoma patients are unknown, we have used SARS-CoV-2 proteins of the original Wuhan, China strain (GenBank: NC_045512). The crystal structures of human CD30/TNFRSF8 (NP_001234.3), CD15/FUT4 (NP_002024.1), SARS-CoV-2 membrane (M) protein (YP_009724393.1), and LMP-1 (YP_401722.1) of EBV are unavailable. It is likely that the inability to crystallize CD30, CD15 and LMP-1 of EBV is determined not only by their transmembrane nature but is also associated with high levels of intrinsic disorder predicted in these proteins. Therefore, we used ITASSER (25) and RaptorX web servers (26) to model these proteins. The models were further refined using GalaxyRefine (27), and the stereochemical quality of the protein structures were determined using the PROCHECK tool available at the SAVES v6.0 server (https://saves.mbi.ucla.edu/). Finally, based on residues in the most favoured region of the Ramachandran plot, models were selected for further analysis. The TMHMM Server v.2.0 (http://www.cbs.dtu.dk/services/TMHMM/) was used to determine the transmembrane helices and extracellular sequences of all proteins.

\section{Protein-protein docking}

We performed protein-protein docking using ZDOCK (28) and HDOCK (29) servers. In most cases, we identified the binding sites/residues from crystal structures using Ligplot+ v.2.2 (30) and the literature. However, when we did not have access to such residue knowledge, we performed blind docking. The top ten complexes from ZDOCK or HDOCK were used for further analysis selecting specific criteria as per the specific objective of the docking (see: Result section). UCSF Chimera (31) was used to visualize and analyze the crystal structures and the docked complexes. 


\section{RESULTS}

\section{D protein models}

Compared to I-TASSER (25), a better model was obtained using RaptorX (26) for human CD30, human CD15, and the SARS-CoV-2 M protein. The RaptorX derived CD30 model was similar to the previously described models $(32,33)$. Therefore, we used this structure of CD30 for protein-protein docking after refinement using GalaxyRefine (27). The Ramachandran plot of the refined CD30 structure (Fig 1A) showed that $87.0 \%$ of the residues are in the most favourable regions. TMHMM analysis suggested that residues 1 to 385 of the CD30 are exposed to the exterior of the cell and therefore, this area was used in docking experiments. For CD15, the final modelled structure (Fig 1B) showed a Ramachandran plot with $92.0 \%$ residues in the most favourable regions. Residues 171 to 530 of CD15 were found on the exterior of the cell as per the TMHMM analysis. RaptorX-derived SARS-CoV-2 M protein when refined with GalaxyRefine (Fig-1C) showed a Ramachandran plot with $92.5 \%$ of residues in the most favorable regions and as per the TMHMM, the residues 1-19 and 74-77 of the M protein were predicted to externally. While we modelled the LMP-1 of the EBV following the same method, $96 \%$ of residues were found in the most favourable regions of the Ramachandran plot. However, this refined structure showed five transmembrane helices. According to a previous report (24), the LMP-1 has six trans-membrane helices, which we achieved directly from the RaptorX. Therefore, we did not use the GalaxyRefine derived structure of LMP-1 for further analysis and docking but used the RaptorX-derived LMP-1 model having six trans-membranes helixes (Fig-1D) that showed $87.7 \%$ of residues in the most favourable regions of the Ramachandran plot. The TMHMM algorithm did not generate any result for LMP-1. Therefore, we considered the membrane topology as described by Kieser, 2007 (24). All these selected 3D models were used for protein-protein docking.

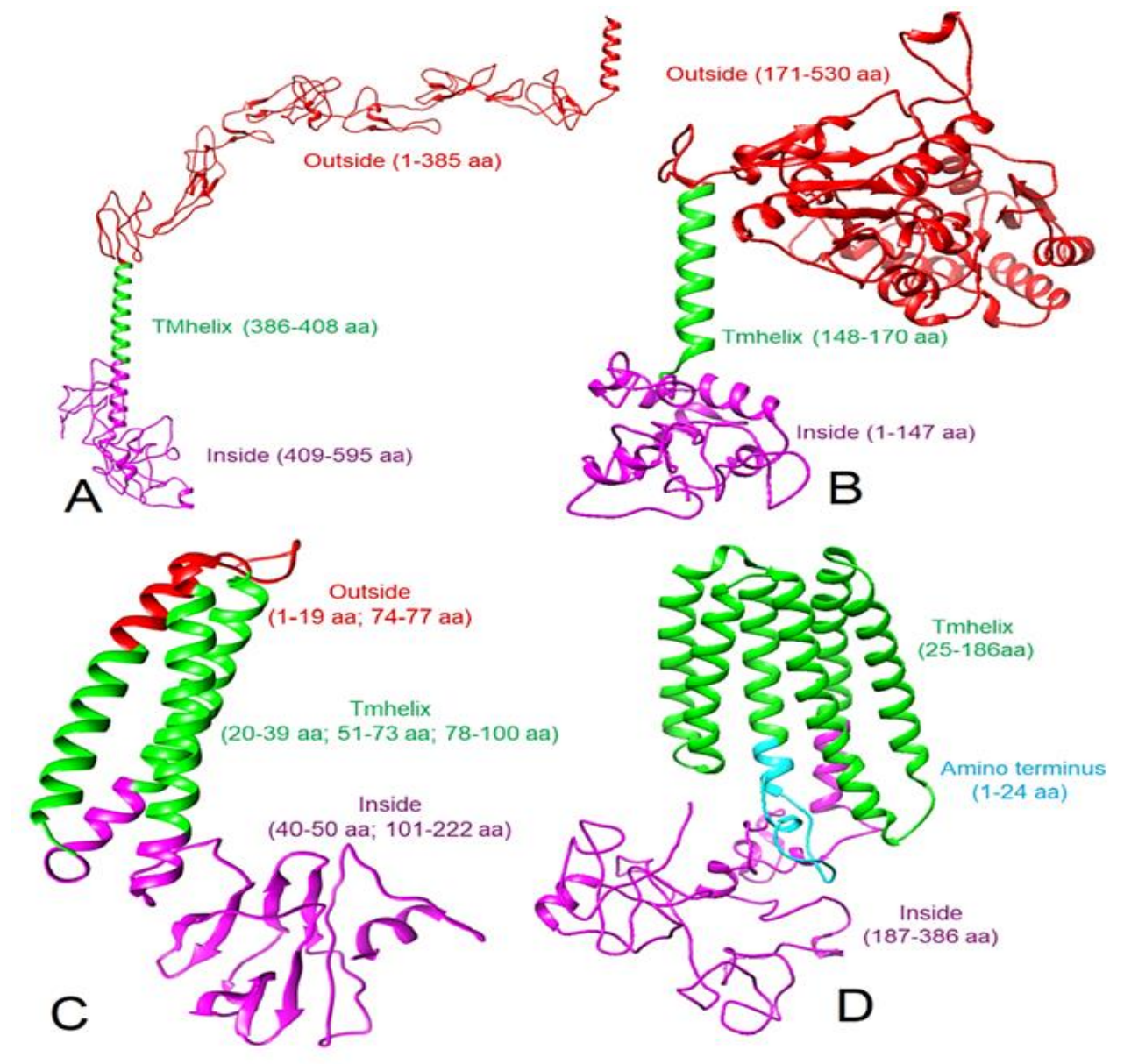

Fig 1: Modelled 3D structures (ribbon view) of proteins and their topology. A) human CD30, B) human CD15, C) SARS-CoV-2 M protein, and D) Epstein-Barr virus LMP-1 protein. 


\section{Spike-RBD may bind to CD15, CD27, CD45, and CD152 receptors of $C H L$ or $F L$}

The first step of SARS-CoV-2 infection is the attachment of the virus to human cells at the human angiotensin-converting enzyme 2 ( $h A C E 2$ ) through its Spike (S) protein receptor-binding domain (RBD), which then facilitates cell membrane fusion for entry into human cells $(34,35)$. To evaluate our hypothesis, we performed protein-protein docking between the Spike-RBD and reported lymphoma cell surface markers (CD15, CD20, CD27, CD30, CD40, CD45, CD80, CD86, CD95, and CTLA-4/ CD152) using ZDOCK server (28).

According to a previous report, the critical residues of Spike-RBD that interact with hACE2 are Lys417, Tyr453, Gln474, Phe486, Gln498, Thr500, and Asn501 (36). However, any peptide that inhibits the interaction of Spike-RBD and hACE2 must bind to three key positions of Spike-RBD (Gly485/Phe486/Asn487, Gln493, and Gln498/Thr500/Asn501) (37). To verify and standardize the ZDOCK or HDOCK based Spike-RBD and human CD receptors docking, we used the crystal structure of the Spike-RBD- $h A C E 2$ complex (PDB: 6LZG) and characterized the interacting residues using Ligplot+ v.2.2. The results showed that the Spike-RBD interacts with hACE2 through residues Lys417, Tyr449, Ala475, Asn487, Gly496, Gln498, Thr500, and Gly502 residues forming $10 \mathrm{H}$-bonds. While we combined these results and our additional structure-based analysis, we observed that four regions of the Spike-RBD (i) R1- Lys417, Tyr449, Tyr453, and its adjacent residues, (ii) R2-Gln498 - Tyr502, (iii) R3-Gln474, Ala475, Phe486, Asn487 (iv) R4-Gly496, Gln493 residues could be critical for strong interaction with any receptor. $\mathrm{R} 2$ and $\mathrm{R} 3$ are probably the most essential regions .

According to the ZDOCK results, the cell exposed domain of CD15 (171-530 aa) potentially interacts with the spike-RBD residues creating $13 \mathrm{H}$-bonds in complex 3 involving its predicted four regions (R1- Tyr449, Asn450, Tyr453; R2- GIn498, Thr500; R3- Asn487; R4- Ser494). In complex 4, the RBD binds to CD15, forming $11 \mathrm{H}$-bonds covering all four regions (R1- Tyr449; R2- Gln498, Asn501, R3Tyr473, Asn487; R4- Gln493, Gly496). In complex 6, the RBD also interacts with CD15 involving four regions (R1-Lys417, Tyr449; R2-Gly502; R3-Glu484; R4-Gly496) and forming $9 \mathrm{H}$-bonds (Fig 2A). ZDOCK did not provide any good result for CD20 (CD antigen 20 or membrane-spanning 4-domains subfamily A member 1, MS4A1) and Spike-RBD docking, and since the IRF4/MUM1 is a nuclear protein, we excluded this $\mathrm{CHL}$ marked from this analysis. Spike-RBD interacts with $\mathrm{CD}$ antigen 30/tumor necrosis factor receptor superfamily member 7 (CD27/TNFRSF7, extracellular domain $20-191$ aa) using four binding regions in complex 8 (R1- Lys417, Tyr449, Tyr453; R2-Gln498; R3-Ala475; R4-GIn493) and in complex 9 (R1-Lys417, Tyr453; R2-GIn498, Thr500; R3-Asn487; R4-Gln493) (Fig 2B). Complex 8 and complex 9 forms respectively 10 and $10 \mathrm{H}$-bonds. A maximum $11 \mathrm{H}$-bonds may be formed between Spike-RBD and the cell surface-exposed (1 to 385aa) residues of human CD30 (complex-5). In this complex, Spike-RBD may bind to CD 30 in two regions (R1-Tyr453; R4-Gln493), and in complex 3, it binds again in two regions (R1-Lys417; R2-Thr500) but forms total $5 \mathrm{H}$-bonds with three other residues. Therefore, CD30 may not be a good candidate to act as a receptor for the S-RBD. S-RBD possibly interacts with maximum $10 \mathrm{H}$-bonds in two complexes with the extracellular domain of CD antigen 40/ Tumor necrosis factor receptor superfamily member 5 (CD40/TNFRSF5, residues 21-193). In complex 8, it covers three regions (R1- Lys417, Tyr449, Tyr453; R2-Gln498; R3- Ala475), and in complex 9, it interacts with four regions (R1- Lys417; R2- Gln498, Thr500; R3- Asn487; R4-Gln493). Although in complex 9 the Spike-RBD is using all four regions to interact with CD40, we did not see any other complex involving all the four regions. Therefore, CD40 also may not be a good receptor for SARS-CoV-2 Spike. In ZDOCK docking, the Spike RBD interacts with CD45 (CD antigen 45 or receptor-type tyrosineprotein phosphatase C, PTPRC) extracellular domain (26- 577 aa) with maximum $11 \mathrm{H}$-bonds in complex-3 where Spike RBD involves four binding regions, each of with one residue (R1- Lys417; R2GIn498; R3- Asn487; R4-GIn493). In complex 8, Spike RBD also interacts using four regions forming $8 \mathrm{H}$ bonds and using 6 residues (R1- Lys417, Tyr453; R2- Gln498, Asn501, R3-Asn487; R4- Gln493) (Fig 2C). Therefore, CD45 could be a good candidate for S-RBD interaction. For CD80 (CD antigen 80 or Tlymphocyte activation antigen CD80) extracellular domain (35-242 aa), the S-RBD forms a total of $9 \mathrm{H}$ bonds in complex 9 and uses three regions (R1- Lys417, Tyr449; R2- Gln498; R4- Gly496) and in complex 3 (total 8 H-bonds) it also involves three regions (R2-Asn501; R3-Ala475; R4-Gln493). Although 
complex 9 involves all the 4 regions, they only make $4 \mathrm{H}$-bonds. Therefore, the Spike-RBD may not also interact with CD 80. When we docked the Spike-RBD with the extracellular domain of CD86 (CD antigen 86 or T-lymphocyte activation antigen CD86), (24-247 aa), we found that the RBD may bind through only three regions (R1- Lys417, Tyr453; R2- Asn501, R4- Gly496) in complex 2 that forms total $10 \mathrm{H}$-bonds. In complex 9, the Spike-RBD forms $8 \mathrm{H}$-bonds; however, it uses three regions (R1-Tyr449; R2-GIn498, Thr500; R4- GIn493). Therefore, CD86 also may not be a good candidate that can act as a receptor for SARS-CoV-2 attachment to human cells. In CD95 (CD antigen 95 or Tumor necrosis factor receptor superfamily member 6 or Apoptosis-mediating surface antigen FAS) extracellular domain (26-173 aa) and S-RBD docking, we observed two complexes that show good interactions involving three regions. In complex 2, the RBD involves R1-Lys417, Tyr449; R3- Asn487; R4-Gln493 and in complex 10, the RBD uses R1-Thr449; R2- Gln498, Thr500; R3-Asn487. the Spike-RBD interacts with our last tested receptor CTLA4/CD152 (Cytotoxic T-lymphocyte protein 4 or CD antigen 125) extracellular domain (36-161 aa) using all four regions in complex 2, where the RBD interacts using residues (R1-Tyr449; R2- Gln498; R3Asn487; R4-Gln493, Gly496) forming $11 \mathrm{H}$-bonds. Further, in complex 3, the RBD involves three key regions (R1-Gly449, Tyr453; R2-Gln498, Asn501, Thr500; R4-Ser494) to interact with CD152 forming 10 $\mathrm{H}$-bonds (Fig 2D). Therefore, CD152 may interact with Spike-RBD and facilitate SARS-CoV-2 attachment and infection.

Therefore, considering the total number of $\mathrm{H}$-bonds and maximum interacting regions and residues of the Spike-RBD, we predicted that CD15, CD27, CD45, and CD152 are top candidates that may act as receptors for the SARS-CoV-2 $S$ protein and can facilitate virus attachment and its subsequent entry into the $\mathrm{CHL}$ cells (Fig 2A-D).
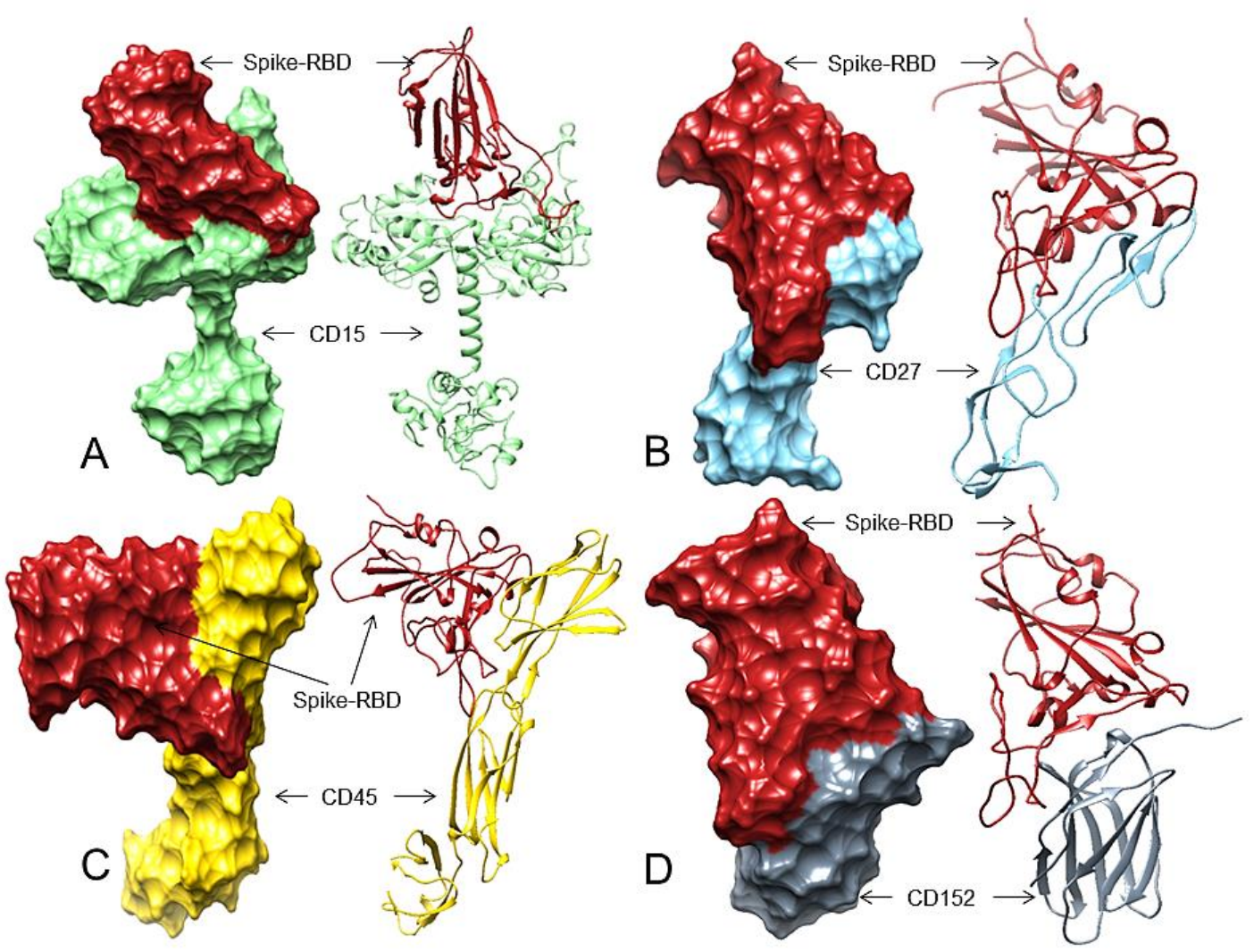

Fig 2: 3D structures (surface and ribbon views) of human CDs and Spike-RBD interactions. A) SpikeRBD (fire brick) binding to human CD15 (light green) (corresponding to complex 3, $13 \mathrm{H}$-bonds, involving four regions of Spike-RBD, B) Spike-RBD (fire brick) binding to human CD27 (sky blue) (corresponding to complex 9, $10 \mathrm{H}$-bonds, involving four regions of Spike-RBD, C) Spike-RBD (fire brick) binding to human CD45 (gold) and Spike-RBD (corresponding to complex 8, $8 \mathrm{H}$-bonds, involving four regions of SpikeRBD, and D) Spike-RBD (fire brick) binding to human CD152 (slate grey) and Spike-RBD (corresponding to complex 2, $11 \mathrm{H}$-bonds, involving four regions of Spike RBD). 

components and may inhibit tubulin nucleation

Next, we focused on identifying if any SARS-CoV-2 protein interacts with the tubulin complexforming components and blocks the microtubule formation to arrest the cell cycle. According to Gordon et al. (38), the SARS-CoV-2 M protein interacts with the human gamma-tubulin complex component 2 (TUBGCP2/ GCP2) and gamma-tubulin complex component 3 (TUBGCP3/GCP3). Similarly, Chen et al, reported that SARS-CoV-2 Orf3a potentially interacts with GCP2, GCP3, and even gamma-tubulin (TUBG1) (39). However, in none of the cases, the authors have provided any further details about the binding sites and the effect of these interactions.

During microtubule (MT) polymerization, the gamma-tubulin ring complex is initially formed as a gamma-tubulin small complex. In this process, each of the GCP2 and GCP3 molecules recruits one TUBG1 molecule at its top and then forms a heterodimer binding through their lateral positions to start the MT nucleation process. Alpha- and beta-tubulin form heterodimers, and through alpha-tubulin, these heterodimers bind to gamma-tubulin. The other GCPs (GCP4, 5, and 6) also follow the same process, and finally, the microtubule polymerization starts $(40,41)$.

We hypothesized that if $\mathrm{M}$ or Orf3 proteins inhibit MT nucleation to restrict the cell cycle, it should bind to any of these three possible areas: (i) the sites where the GCP2 and GCP3 bind laterally to form heterodimers (ii) the gamma-tubulin binding sites of GCP2 or CGP3, and (iii) the TUBG1 sites that are used to interact with GCP2 or GCP3.

To test this hypothesis, we first identified the binding residues between GCP2 and GCP3. For this purpose, we used two crystal structures: the human GCP2 - GCP3 complex (PDB: 6V6B) and the human gamma-tubulin ring complex (PDB: 6V6S). Next, we standardized the residue positions of PDB: 6V6S corresponding to PDB: $6 \mathrm{~V} 6 \mathrm{~B}$, and we noted the interacting residues in the GCP2 - GCP3 complex according to PDB: 6V6B using Ligplot+ v.2.2. We identified the interacting residues between GCP2GCP3 at lateral positions as GCP2: Ser283, Gly396, Glu242, Gln350; GCP3: Arg252, Ser323, His343, GIn529 that form $3 \mathrm{H}$-bonds and one salt bridge (Fig $3 \mathrm{~A}$ ). We found that the chains $\mathrm{C} / \mathrm{G}$ (GCP2) and B/F (GCP3) of PDB: 6V6S show similar interaction as the interactions found in PDB: 6V6B.

Since there is no crystal structure for GCP2-TUBG1 and GCP3-TUBG1, we identified the interactions between TUBG1-GCP2 and TUBG1-GCP3 the gamma-tubulin ring complex (PDB: 6V6S) using Ligplot+ v.2.2 and considered that at least two complexes in this ring complex should show similar interactions. Furthermore, the corresponding residues of GCP3 and TUBG1 were identified in PDB: 6V6B and PDB: 6V5V through structural overlapping using UCSF Chimera. A similar approach was applied to identify GCP2 and TUBG1 interacting residues. We observed that GCP2 (chains C/G) (PDB: 6V6B) interacts with TUBG1 (PDB: 6V5V) using $10 \mathrm{H}$-bonds and two salt bridges (TUBG1: Arg3, Arg47, Tyr248, Ile254, Ser259, Pro262, Asp329, Pro353, Ala354, Gln357; GCP2: Asp554, Asp561, Cys684, Arg711, Asn716, Gln719, Asn720, Glu731, His735, Asn890) and GCP3 (chains B/F) (PDB: 6V6S) interacts with TUBG1 (PDB: 6V5V) using $14 \mathrm{H}$-bonds and three salt bridges (TUBG1: Arg3, Thr45, Arg47, Pro162, Lys163, Asn198, Tyr248, Asp252, Pro265, His334, Arg341, Trp351, Ser355, Gln357; GCP3: Asp572, Arg575, Lys671, Asp579, Asn609, Arg681, Lys682, Cys686, Lys689, Ser709, Gln717, Gln719, Glu725, Glu884, His885).

\section{M and Orf3a proteins may interact at GCP2-GCP3 binding sites}

To explore if the $M$ and Orf3a proteins hinder the lateral binding of GCP2 and GCP3, we selected the GCP2 (GCP2: Ser283, Gly396, Glu242, Gln350) and GCP3 (GCP3: Arg252, Ser323, His343, Gln529) interacting residues and docked them with the $M$ and Orf3a proteins using the HDOCK server. Among the four residues of GCP2 that form $3 \mathrm{H}$-bonds with GCP3, the $\mathrm{M}$ protein may interact with two residues (Ser283 and Gln350) along with two nearby residues forming $6 \mathrm{H}$-bonds (complex-7) (Fig 3B). In complex 3, GCP2 interacts with the M protein forming $9 \mathrm{H}$-bonds (Thr235, Ser243, Arg290, Ser393). However, the residues are not the same as it uses to interact with GCP3. When we performed the docking between SARS-CoV-2 M protein with GCP3 targeting its GCP2 binding residues, only one residue (Arg252) of 
GCP3 interacted in three different complexes (complex-4, -6, -7). However, other interacting residues of GCP3 are not also close to the mapped interacting residues involved in GCP2-GCP3 interaction (.

We performed a similar analysis with Orf3a as we did for M protein. Although Orf3a interacts with GCP2 with a maximum of $7 \mathrm{H}$-bonds (complex 4), it does not involve any residue of GCP2 that interacts with GCP3. On the other hand, Orf3a potentially interacts with GCP3 at its GCP2 binding site. In complex 7 it makes $5 \mathrm{H}$-bonds, and one salt bridge involves 2 residues (out of 4) of GCP3 (Ser323, Arg252, His343-salt bridge) that are involved in lateral binding with GCP2 and other 2 residues (Gln322, Arg333) (Fig $3 \mathrm{C}$ ). In complex 3 it makes $7 \mathrm{H}$-bonds and involves 7 residues of GCP3; however, only one residue (Arg252) interacts with GCP2.

Taken together, the M protein may not bind to GCP3 at its GCP2 interacting site. However, the M protein possibly interacts with GCP2 at its GCP3 binding site using 2 residues (out of 4) (Fig-3A,B). On the other hand, Orf3a may interact with GCP3, blocking at least 3 (out of 4) of its GCP2 binding residues (Fig 3A,C). Therefore, the potential interactions of M-GCP2 or Orf3a-GCP3 may affect the GCP2-GCP3 lateral binding and hinder microtubule nucleation.

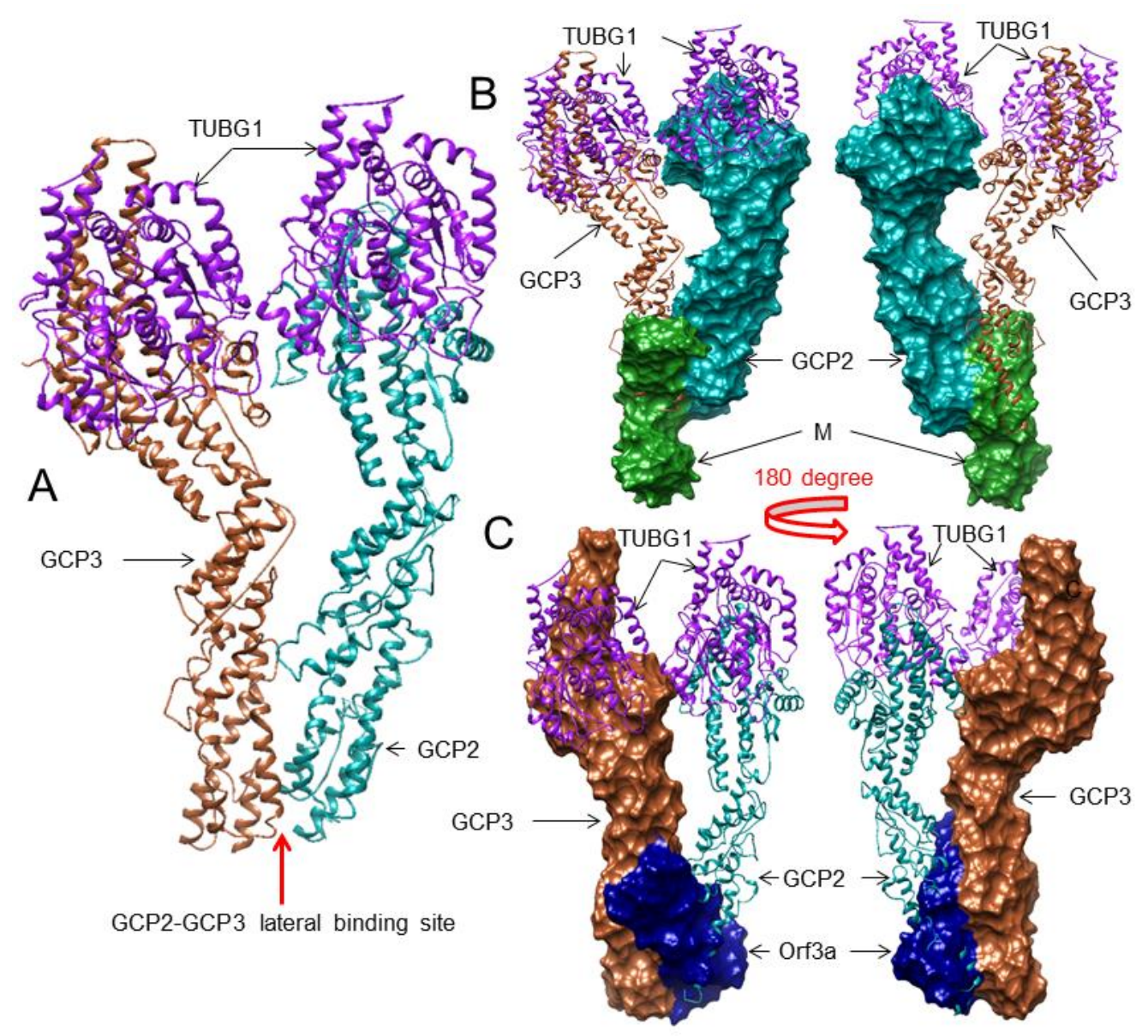

Fig 3: 3D structures (surface and superimposed views) of $M$ protein and Orf3a's interactions with GCP2-GCP3 complex. A) The lateral binding positions of GCP2 (dark cyan) and GCP3 (sienna) in native human gamma-tubulin ring complex crystal structure (PDB: 6V6B. B) Binding of $\mathrm{M}$ protein (forest green) to GCP2 (dark cyan) at its GCP3 binding lateral position (front and 180-degree rotation views, corresponding to complex 7, C) Binding of Orf3a (navy blue) to GCP3 (sienna) at its GCP2 binding lateral position (front and 180-degree rotation views, corresponding to complex 7).

\section{Both the M and Orf3a proteins may interact with GCP3 at its TUBG1 binding sites}

To understand if $M$ and Orf3a proteins interact with TUBG1, we docked both proteins at four different positions: (i) TUBG1 binding sites/residues of GCP2, (ii) TUBG1 binding sites/residues of GCP3, (iii) GCP2 binding sites/residues of TUBG1, and (iv) GCP3 binding sites/residues of TUBG1. 
Out of 10 GCP2 residues that interact with TUBG1, the M protein can maximally bind to 3 such GCP2 residues (Asp554, Arg711, Asp561) and 2 additional residues (Tyr723, Ser885) in complex 5 forming $6 \mathrm{H}$-bonds. In complex 1 , the interactions show $5 \mathrm{H}$-bonds. However, only $3 \mathrm{GCP} 2$ residues (Glu731, Cys684, Ser885) interact with TUBG1. The M protein interacts with GCP3 using maximally 10 H-bonds involving 3 key residues (Lys671, Lys689, Ser709) and 6 other residues of GCP3 in complex 1 (Fig-4A-C). In complex 3, the M protein binds to 3 TUBG1 interacting residues of GCP3 (Asp572, Arg681, Ser709) and 4 other residues (Gly571, Phe612, His716, Asn883), which are close to the TUBG1 interacting residues of GCP3 forming $8 \mathrm{H}$-bonds.

Orf3a binds to the TUBG1 interacting GCP2 residues in 2 complexes (complex 3 and 9), forming $7 \mathrm{H}$-bonds. In complex 9, 7 residues are involved where 4 residues (GIn719, Cys684, Asn716, Asp561) interact with TUBG1. However, in complex 3, only 4 residues of GCP2 interact with ORF3a, where 3 residues (Cys684, Gln719, Asn890) of GCP2 interact with TUBG1. In GCP3 (TUBG1 binding site) -Orf3a interaction, in complex 1 GCP3 interacts with Orf3a involving 7 residues and $12 \mathrm{H}$-bonds, where 3 residues of GCP3 (Arg681, Ser709, Gln717) interact with TUBG1 (Fig 4D-F). In complex 3 we found $5 \mathrm{H}$ bonds and only 2 such GCP3 residues (Asn609, Gln717) interact with Orf3a.

Therefore, our results suggest that the SARS-CoV-2 M protein may interact with the TUBG1 binding site of GCP3 and probably block GCP3 binding to TUBG1. However, considering the number of $\mathrm{H}$-bonds and the number of GCP residues involved, Orf3 may bind to GCP3 at its TUBG1 binding site more strongly than GCP2. The probable 3D interactions are shown in Fig 4A-F. Since both M and Orf3a are probably interacting with GCP3 at its TUBG1 binding sites, we also performed a superimposition analysis that also shows the M and Orf3a interaction with GCP3 may inhibit TUBG1 binding to GCP3 (Fig $4 G, H)$. Nevertheless, additional analyses are needed if these interactions of the $M$ and Orf3a proteins inhibit TUBG1 binding to GCP3.

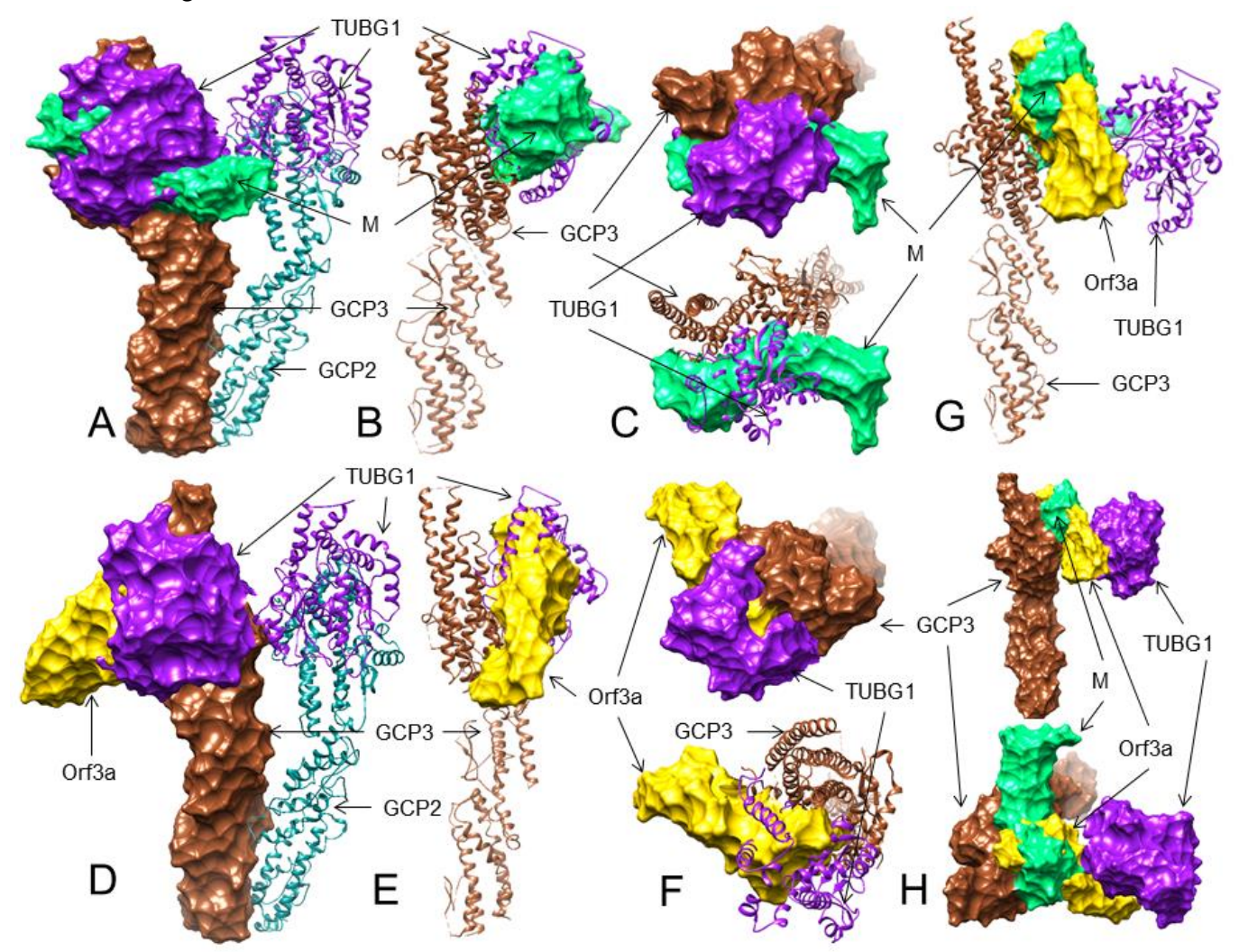

Fig 4: 3D structures (surface, ribbon, and superimposed views) of $M$ protein and Orf3a interactions with GCP3 at its TUBG1 binding site. A) Superimposed (front view) structure showing SARS-CoV-2 M protein (spring green) binding to GCP3 (sienna) at its TUBG1 (purple) binding position blocking GCP3TUBG1 interaction (corresponding to complex 1), B) its lateral view, and C) its top views. D) Front view structure showing SARS-CoV-2 Orf3a (gold) binding to GCP3 (sienna) at its TUBG1 (purple) binding position blocking GCP3-TUBG1 interaction (corresponding to complex 1), E) its lateral view, and F) its top views. G) Superimposed (front view) complex of both $M$ and Orf3a binding to GCP3 at its TUBG1 binding site and $\mathbf{H}$ ) its front and top views. 
In docking studies of the $M$ protein with TUBG1 binding sites that interact with GCP2, no interaction involving the GCP2 binding residue of TUBG1 was detected except complex 1, where only one matching residue (Tyr248) was observed. However, in this model, we found $9 \mathrm{H}$-bonds. Similarly, in complexes 7 and 8 , the M protein interacted with TUBG1 using 8 and $9 \mathrm{H}$-bonds, respectively. For TUBG1 binding sites for GCP3, we got better results. The $\mathrm{M}$ protein can bind with $9 \mathrm{H}$-bonds (complex-1) and involves 5 residues (Tyr248, Asp252, His334, Arg341, Trp351) of TUBG1 (out of 14) that interact with GCP3 (Fig 5A-D). In complexes 8 and 9, the M protein interacts with TUBG1 making 10 and $7 \mathrm{H}$-bonds, respectively. In complex 8 it uses two common residues (Pro162, Arg265), and in complex 9 four common residues (His334, Arg341, Ser355, Gln357).

In Orf3a, although it makes $7 \mathrm{H}$-bonds in complex 3, it does not involve any TUBG1 residue that interacts with GCP2. However, in complex-1, only $4 \mathrm{H}$-bonds are formed by 4 residues, where only 2 residues (Tyr248, GIn357) of TUBG1 are involved in binding with GCP2. In the case of GCP3, we also found the same trend. Out of 16 TUBG1 residues, those interact with GCP3, only 4 of them (Tyr248, His334, Arg341, Gln357) probably bind to Orf3a, forming $4 \mathrm{H}$-bonds in complex-1 (Fig 5E-H). In this complex His334 and Arg341 form H-bonds with Orf3a. However, in TUBG1-GCP3 interaction, these two residues of TUBG1 form salt bridges. In complex 3, a total of 6 residues of TUBG1 (Arg212, Asp216, Val305, Arg343, Arg390, Gln394) bind with Orf3a making $7 \mathrm{H}$-bonds. However, none of these TUBG1 residues interact with GCP3.

Taken together, our analysis suggests that the M protein may bind to TUBG1, blocking its binding to GCP3 more strongly than GCP2. The probable 3D interactions between TUBG1-M are shown in Fig 5A-D. Considering the number and specific residues of TUBG1 involved in GCP2 and GCP3 interactions, it is unlikely that the same residues interact with Orf3a and interfere with TUBG1 binding to GCP2 and GCP3 (Fig 5E-H).
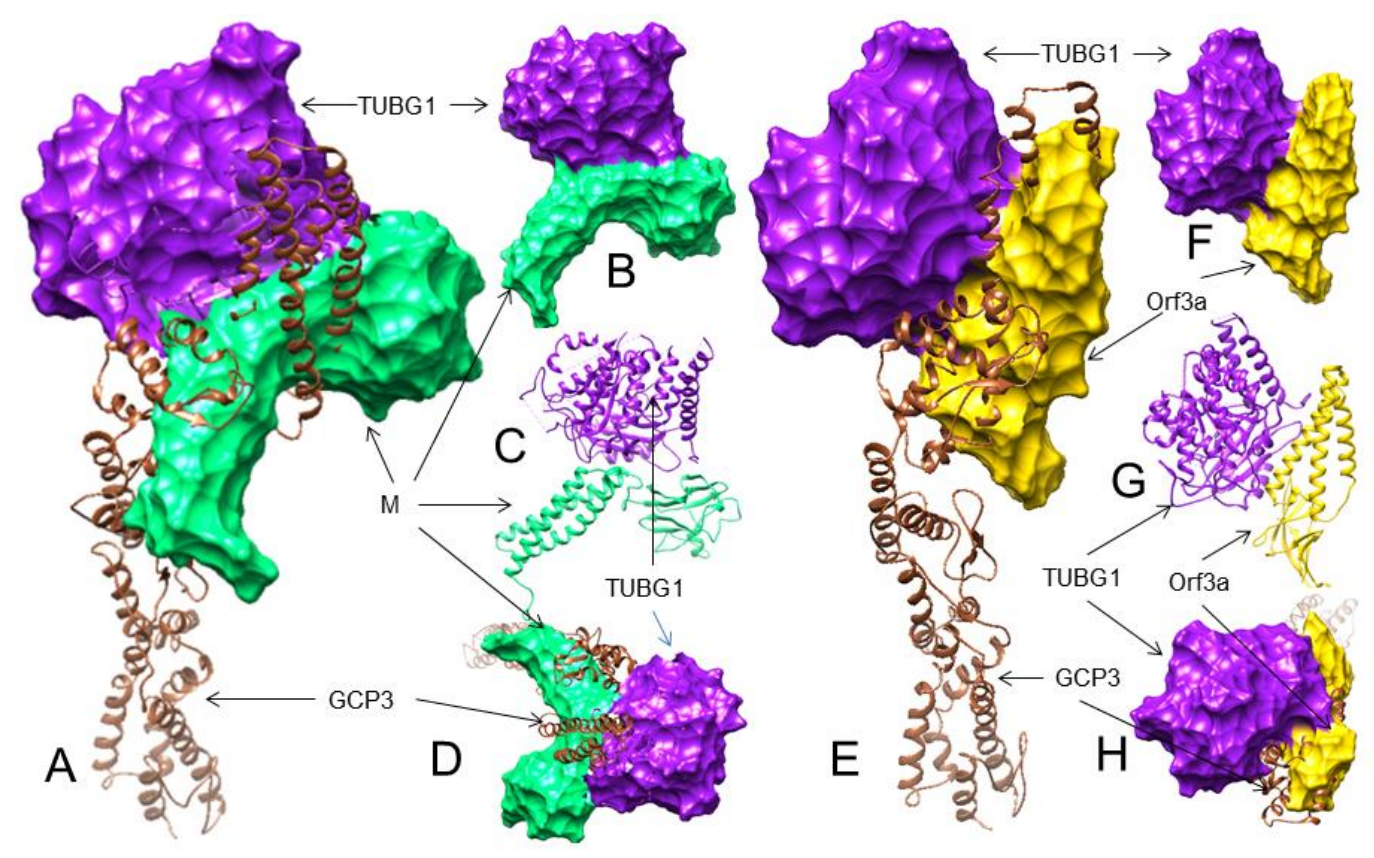

Fig 5: 3D structure (surface, ribbon, and superimposed views) of TUBG1-M and TUBG1-Orf3a interactions at GCP3 binding site of TUBG1. A) Superimposed view of $M$ protein (spring green) binding to TUBG1 (purple) at its GCP3 binding position blocking GCP3-TUBG1 interaction (corresponding to complex 1), B) front surface view, C) front ribbon view, and D) superimposed top view. E) Superimposed view of Orf3a (gold) binding to TUBG1 (purple) at its GCP3 binding position blocking GCP3-TUBG1 interaction (corresponding to complex 1), F) front surface view, G) front ribbon view, and H) superimposed top view. 
The LMP-1 protein of EBV is essential for EBV-associated oncogenesis. Since the cHL patient described by Challenor and Tucker (2) tested positive for EBV, we additionally hypothesized that some SARS-CoV-2 proteins might inhibit the oncogenic signaling of LMP-1 by binding its cytoplasmic signaling motif residues 204-208 aa/ "PQQAT" and 379-384 aa/ "PVQLSY" that recruit TRAFs and TRADD, respectively, to activate the oncogenic NF-kB signaling induced B-cell proliferation (24).

To test this hypothesis, we performed a SARS-CoV-2 proteome-wide docking with our modeled LMP-1 using ZDOCK. In this docking, we selected the LMP-1 residues 202-210 aa (TRAFs binding site) and 375-386 aa (TRADD interacting site) and docked them with SARS-CoV-2 proteins without selecting any residues. We considered few principles in this analysis (i) since the LMP-1 interacting motifs of TRAFs or TRADD are only 4-5 amino acids of length, a SARS-CoV-2 protein must interact with at least two critical amino acids of any of these motifs, and (ii) more than two ZDOCK models should show the same interacting amino acids of TRAFs or TRADD binding motifs with hydrogen bonds.

Based on these criteria, we found that out of 25 SARS-CoV-2 proteins we docked, 7 proteins namely PLpro/nsp3 (GIn381, Ser383, Tyr384, Asp386), 3CLpro/Mpro (Ser383, Tyr384, Tyr385), nsp7 (Gln381, Ser383, Tyr384, Tyr385), nsp10 (Gln381, Ser383, Tyr384, Tyr385), RdRp/nsp12 (Gln381, Ser383, Tyr385, Asp386), Spike (GIn381, Tyr384, Tyr385), and Orf8 (Gln381, Ser383) may potentially block the TRADD interacting motif of LMP-1.

For activation of NF-kB, TRADD binding to the PVQLSY motif of LMP-1 is essential, and mutations in the Tyr384 and Tyr385 residues of LMP-1 stop the TRADD interaction with LMP-1 (24). Considering these facts and our results, it is therefore suggested that 3CLpro/Mpro (Fig 6A), nsp7 (Fig 6B), nsp10 (Fig 6C), and Spike (Fig 6D) proteins of SARS-CoV-2 may interact with the LMP-1 of EBV at its TRADD binding residues and potentially regulate the NF-kB oncogenic signaling for B-cell proliferation in this patient.
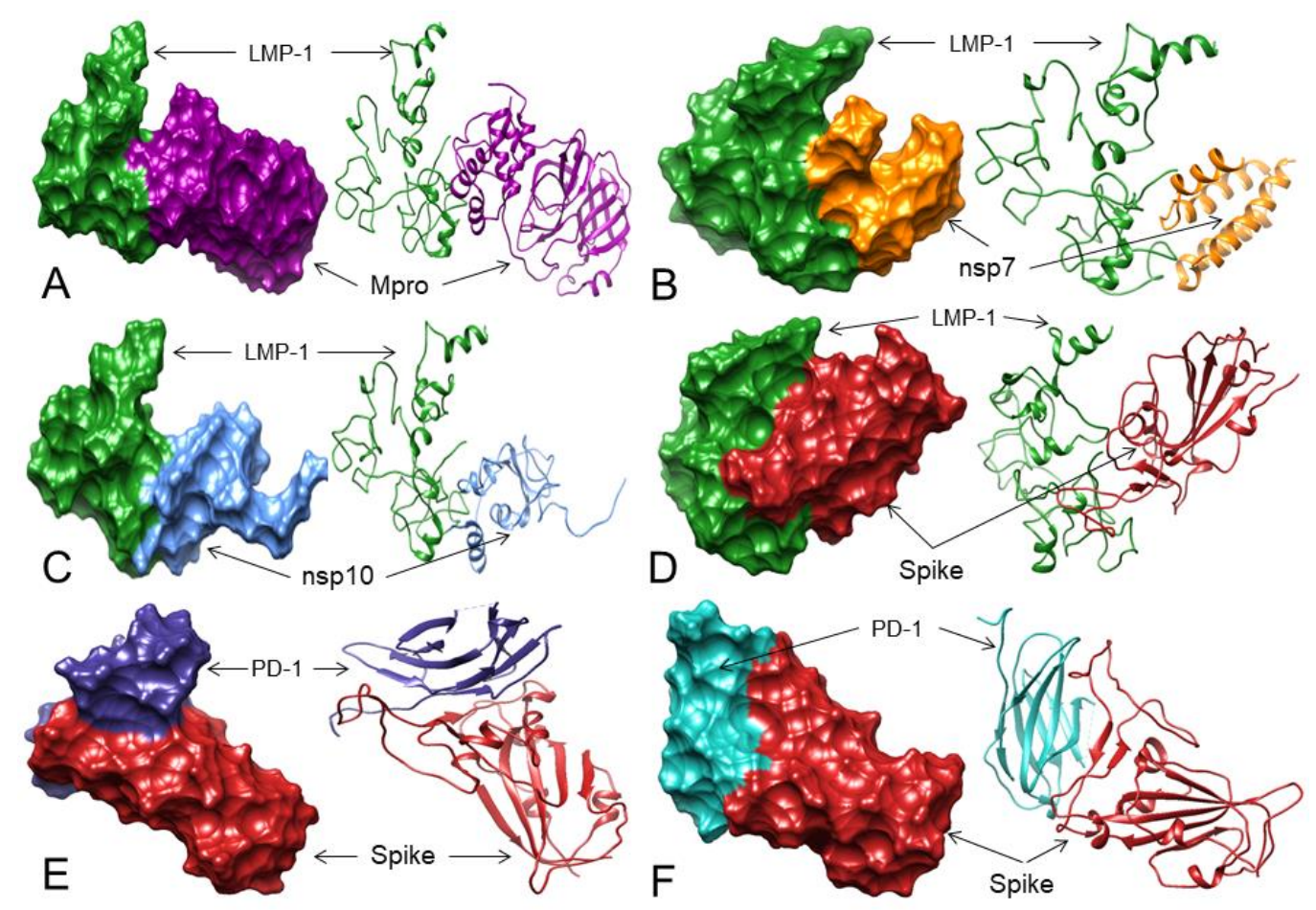

Fig 6: 3D structures (surface and ribbon views) of SARS-CoV-2 proteins binding to LMP-1 and PD1 - Spike RBD interactions. A). 3CLpro/Mpro (dark magenta) binding to LMP-1 (forest green) (corresponding to complex 9, $7 \mathrm{H}$-bonds), B) nsp7 (orange) binding to LMP-1 (forest green) (corresponding to complex 7, $8 \mathrm{H}$-bonds), C) nsp10 (cornflower blue) binding to LMP-1 (forest green) (corresponding to complex 2, $9 \mathrm{H}$-bonds), and D) Spike (fire brick) binding to LMP-1 (forest green) 
(corresponding to complex 6, $5 \mathrm{H}$-bonds). E) Spike-RBD (fire brick) binding to PD-1 (dark slate blue) at its nivolumab binding sites (corresponding to complex 2, $8 \mathrm{H}$-bonds) and F) Spike-RBD (fire brick) binding to PD-1 (dark slate blue) at its pembrolizumab binding sites (corresponding to complex $8,10 \mathrm{H}$-bonds).

\section{Does the Spike protein interact with PD-1 to block access to PD-L1 and PD-L2?}

Finally, we checked if the SARS-CoV-2 Spike protein interacts with PD-1 and acts similarly to the T-cell checkpoint inhibiting monoclonal antibodies (mAbs) like nivolumab and pembrolizumab to induce PD-1 mediated apoptosis.

To check the feasibility of this hypothesis, we first mapped the binding residues between PD-1 and these two mAbs from PD-1 in complex with pembrolizumab Fab (PDB: 5GGS) and PD-1 in complex with nivolumab-Fab (PDB: 5WT9) through literature search $(42,43)$. It was found that pembrolizumab-Fab interacts with PD-1 residues (Phe63, Asn66, Thr76, Lys78, Glu84, Ser87, Gly90, Lys131, Ala132) and nivolumab-Fab binds to PD-1 residues (Leu25, Ser27, Pro28, Asp29, Arg30, Thr59, Ser60, Leu128, Ala129, Prp130, Lys131, Ala132). After this mapping, we performed Spike-RBD (without selecting any residue) and PD-1 (selecting residues that interact with nivolumab and pembrolizumab) docking using ZDOCK. Although no interaction of Spike-RBD was detected with PD-1, the complex-2 that forms $8 \mathrm{H}$ bonds showed that RBD could interact with PD-1 near the stretch of nivolumab interacting residues of PD-1 (Fig 6E). On the other hand, complexes 7 and 8 form 6 and $10 \mathrm{H}$-bonds, respectively, where the RBD may interact with the stretch of pembrolizumab interacting residues of PD-1 (Fig 6F).

Although the Spike-RBD does not bind exactly to the pembrolizumab and nivolumab interacting residues of PD-1, it may interact with PD-1 at very close proximity to the PD-1 residues that interact with these two mAbs. Therefore, the Spike-RBD may bind to PD-1 and may act as pembrolizumab and nivolumab-like mAbs to block access to PD-L1 and PD-L2 leading to cHL cell apoptosis and remission.

\section{DISCUSSION}

During the initial days of COVID-19 pandemic, there were no reports of any direct correlation between SARS-CoV-2 and lymphoma. However, in December 2020, based on a multi-omics approach, we predicted that lymphoma would have an association with COVID-19 $(44,45)$. In January 2021, Challenor and Tucker first brought to our attention that a 61-year-old man with $\mathrm{cHL}$ and tested positive for EBV showed complete remission of $\mathrm{cHL}$ after he was infected with SARS-CoV-2 (2). The second case was reported by Sollini et al. in February 2021, where a 61-year-old patient suffering from FL showed complete remission upon SARS-CoV-2 infection (3). Challenor and Tucker suggested that this SARSCoV-2 may induce an unknown specific anti-tumor immune response mechanism (44) that may also be responsible for a previously reported case of spontaneous regression of diffuse large B-cell lymphoma (DLBCL) after infection with Clostridium difficile (46). Similarly, Sollini et al (46) suggested that the SARSCoV-2 induced remission of their reported FL case was probably due to a "flare phenomenon" as observed in immunotherapy that finally results in an "abscopal effect". A recent report in May 2021 from Spain also suggested that SARS-COV-2 triggers an anti-tumor immune response in lymphoma (47). In addition, a 22-year-old Hodgkin lymphoma patient suffering from COVID-19 was successfully treated with the PD-1 inhibitor pembrolizumab (48), and the $\mathrm{cHL}$ was generally treated with CD30 targeting brentuximab vedotin (16). Therefore, unlike the anti-tumor mechanisms of other oncolytic single-stranded RNA viruses (4,6-9), we also focused on possible anti-tumor immune response by SARS-CoV-2 in the reported $\mathrm{cHL}$ or $\mathrm{FL}$ cases.

As per our results, the initial contact and attachment of SARS-CoV-2 to cHL or FL cells may be possible through the interaction between the SARS-CoV-2 Spike-RBD and cell surface markers for $\mathrm{cHL}$ or FL such as CD15, CD27, and CD152 (Fig 2A-D). SARS-CoV-2-induced remission of CHL and FL are isolated cases $(3,44)$ compared to $\mathrm{CHL}$ and FL cases globally and the magnitude of COVID-19 pandemic. Previous reports suggest that some unclassified FL expresses CD15 (49) and CD27 (50). Additionally, the anti-CD152 mAb ipilimumab could be a potential targeted therapeutic for FL (51). Similarly, CD15 has been reported to be expressed in some specific groups of $\mathrm{CHL}$ patients (52), circulating blood cells of $\mathrm{cHL}$ 
patients show clonal expression of CD27 (53), and expression of CD152 is also reported in $\mathrm{CHL}(10,54)$. Therefore, these two patients $(3,44)$ may express very specific receptors like CD15, CD27, and CD152 that are not commonly expressed in cHL and FL, and the SARS-CoV-2 Spike-RBD may use any of these receptors to enter into the lymphoma cells.

As per our hypothesis, SARS-CoV-2 infection leads to lymphoma remission using a similar mechanism as the brentuximab vedotin exhibits; upon entry into the lymphoma cells, the SARS-COV-2 it will use some of its proteins to initiate cell cycle arrest or apoptosis through interacting with cell cycle machinery components. Gordon et al. (38) and Chen et al, (55) reported that M protein and Orf3a of SARS-CoV-2 interact with gamma-tubulin complex components GCP2, GCP3, and TUBG1. The binding of GCP2-TUBG1, GCP3-TUBG1, and GCP2-GCP3 is essential for the microtubule nucleation process $(40,41)$. Our analysis suggests that the M protein may bind to GCP2 at its GCP3 binding site (Fig 3B), and Orf3a may bind to GCP3 at its GCP2 binding residues (Fig 3C); thus, both the M and Orf3a proteins can potentially render the GCP2-GCP3 lateral binding. Further, the $M$ and Orf3a proteins may also bind to GCP3 at its TUBG1 binding site (Fig $4 \mathrm{~A}-\mathrm{H}$ ). Additionally, we also predicted that the $\mathrm{M}$ and Orf3a proteins might bind to TUBG1 blocking its interaction with GCP3 (Fig 5A-H). Therefore, if the M and Orf3a proteins are involved in such interactions, the microtubule nucleation process will stop leading to cell cycle arrest.

Some coronavirus infection induces apoptosis in host cells that may be required for the viral replication and propagation in respective hosts (56). SARS-CoV and SARS-CoV-2 induce apoptosis through distinct mechanisms (57). SARS-CoV uses the 7a protein to induce apoptosis, activating the p38 MAPK pathway (58). SARS-CoV also uses the $S, M$, and $N$ proteins for induction of apoptosis $(59,60)$. Recently, the SARS-CoV-2 Orf3a was reported to induce apoptosis via the release of cytochrome C (61). As per our prediction, Orf3a may also be involved in cell cycle arrest at a very early stage, leading to apoptosis or cell death (Fig 4D-F, Fig 5E-H). PD-1 immune checkpoint blocking mAbs such as pembrolizumab and nivolumab are used in $\mathrm{CHL}(19,20)$ and $\mathrm{FL}(18)$ to induce apoptosis. Although we do not know if the reported $\mathrm{CHL}$ and FL cases $(3,44)$ are PD-1 positive, we analyzed whether SARS-CoV-2 Spike may act like pembrolizumab and nivolumab binds to PD-1 to block the access of PDL-1 and PDL-2 and thus down-regulate PD-1 signaling and induce apoptosis. Our analysis observed that the Spike-RBD potentially interact with PD-1 to the binding sites of these two mAbs, although only a few overlapping residues of PD-1 that interact with pembrolizumab nivolumab are found to interact with Spike-RBD (Fig $6 \mathrm{E}, \mathrm{F})$. Therefore, we suggest further validating whether the SARS-CoV-2 Spike protein can also induce apoptosis through negative regulation of PD-1 mediated signaling.

Our previous report predicted that the SARS-CoV-2 infection pathway is involved in crosstalk with other viral pathways, including EBV (62). Co-infection of EBV in COVID-19 patients is not uncommon, and in COVID-19 patients, reactivation of EBV has been reported, which may be associated with disease severity and other symptoms of long COVID-19 (63-66). Additionally, patients with lymphoproliferative disorders showing immunodeficiency and post-transplantation patients subjected to immunosuppression, the synergistic action of EBV and SARS-CoV-2 may increase the fatality rate (67). The CHL patient of Challenor and Tucker was EBV positive (2), and LMP-1 of EBV is the main oncogenic protein of EBV that activates oncogenic signaling through activation of NF-KB, JAK/STAT, and PI3K/AKT pathways through its cytoplasmic TRAFs and TRADD binding motifs $(21,24)$. Contrary to the reported potential synergistic association between EBV and SARS-CoV-2 (63-67), in our analysis, we found that 3CLpro/Mpro, nsp7, nsp10, and S (Fig 6A-D) proteins of SARS-CoV-2 may bind to the TRADD binding sites of LMP-1 and this binding may regulate NF-kB oncogenic signaling. However, we are not sure if this interaction negatively or positively regulates the oncogenic signaling; therefore, it warrants further investigation.

\section{CONCLUSION}

This study tried to explore the possible molecular mechanism behind the rare phenomenon of lymphoma remission upon SARS-CoV-2 infection. We have focused on a specific anti-tumor immune response by SARS-CoV-2. We are currently planning to conduct in vitro experimental validations of our results and if our proposed mechanism is proven through these experiments, the SARS-CoV-2 may be engineered for effective therapeutic interventions against lymphoma and other proliferative disorders, 


\section{Abbreviations}

hACE2

BSAP

CD

CD15

CD20

CD27

CD30

CD40

CD45

CD80

CD86

CD95

CD125

$\mathrm{CHL}$

COVID-19

CTLA4

DLBCL

EBV

FL

FUT4

GCP2

GCP3

GGT1

HRS

IRF4

JAK

mABs

LMP-1

$M$

MMAE

MS4A1

MT

PAX5

MUM1

PD-1

PD-L1

PD-L2

PTPRC

SARS-CoV-2

RBD

$S$

STAT

TNFRSF5

TNFRSF6

TNFRSF7

TNFRSF8

TRAFs

TRADD

TUBG1
Human Angiotensin-converting enzyme 2

B-cell-specific transcription factor

Cluster of differentiation

CD antigen 15 (alpha-(1,3)-fucosyltransferase 4, FUT4)

CD antigen 20 (B-lymphocyte antigen CD20)

CD antigen 27 (Tumor necrosis factor receptor superfamily member 7 , TNFRSF7)

CD antigen 30 (tumor necrosis factor receptor superfamily member 8 , TNFRSF8)

CD antigen 40 (Tumor necrosis factor receptor superfamily member 5 , TNFRSF5)

CD antigen 45 (Receptor-type tyrosine-protein phosphatase C, PTPRC)

$\mathrm{CD}$ antigen 80 (T-lymphocyte activation antigen CD80)

$\mathrm{CD}$ antigen 86 ( $\mathrm{T}$-lymphocyte activation antigen CD86)

CD antigen 95 (Tumor necrosis factor receptor superfamily member 6 , TNFRSF6, or Apoptosis-mediating surface antigen FAS)

CD antigen 125 (Cytotoxic T-lymphocyte protein 4, CTLA4)

Classical Hodgkin lymphoma

Coronavirus Disease 2019

T-lymphocyte associated protein 4 (CD antigen 125, CD125)

Diffuse large B-cell lymphoma

Epstein-Barr virus

Follicular lymphoma

Alpha-(1,3)-fucosyltransferase 4 (CD antigen 15, CD15)

Gamma-tubulin complex component 2

Gamma-tubulin complex component 3

Gamma-glutamyl transferase 1

Hodgkin or Reed-Sternberg cells

Interferon regulatory factor 4

Janus kinase 1

Monoclonal antibodies

Latent oncogenic membrane protein 1

Membrane protein of SARS-CoV-2

Monomethyl auristatin E

Membrane-spanning 4-domains subfamily A member 1

Microtubule

Paired box protein Pax-5

Multiple myeloma oncogene 1

Programmed cell death-1

Programmed cell death 1 ligand 1

Programmed cell death 1 ligand 2

Receptor-type tyrosine-protein phosphatase C (CD antigen 45, CD45)

Severe acute respiratory coronavirus 2

Receptor binding domain of spike protein

Spike protein of SARS-CoV-2

Signal transducer and activator of transcription

Tumor necrosis factor receptor superfamily member 5 (CD antigen 40, CD40)

Tumor necrosis factor receptor superfamily member 6 (CD antigen

95,CD95)

Tumor necrosis factor receptor superfamily member 7 (CD antigen 27 , CD27)

Tumor necrosis factor receptor superfamily member 8 (CD antigen 30)

Tumor necrosis factor receptor-associated factors

Tumor necrosis factor receptor type 1-associated DEATH domain protein

Tubulin gamma-1 chain 


\section{Author contribution}

DB: Conceptualization, methodology, design of the research, and project management; DB, ST, LGRM, VNU: data collection and analysis; DB, ST, MEW, KJA, BSA, KFA: result interpretation, DB: wrote the paper; AAAA, VNU, KL, SSH, AS-A, KT, PG, EMR, SCS, VA, TM: editing and technical inputs. All authors read and approved the article for publication.

\section{Funding}

No financial support was received for this research.

\section{Acknowledgement}

VA, ST, LGRM are supported by CAPES, FAPEMIG, the state foundation and CNPq federal agency. KJA acknowledges the Taif University Researchers Supporting Program (project number: TURSP-2020/128), Taif University, Saudi Arabia.

\section{Conflict of interest}

Authors declare no conflict of interest.

\section{REFERENCES}

1. Jarahzadeh, M.H., Asadian, F., Farbod, M., Meibodi, B., Abbasi, H., Jafari, M., Raee-Ezzabadi, A., Bahrami, R. and Neamatzadeh, H. (2021) Cancer and Coronavirus Disease (COVID-19): Comorbidity, Mechanical Ventilation, and Death Risk. Journal of gastrointestinal cancer, 52, 8084.

2. Challenor, S. and Tucker, D. (2021) SARS-CoV-2-induced remission of Hodgkin lymphoma. British journal of haematology, 192, 415 .

3. Sollini, M., Gelardi, F., Carlo-Stella, C. and Chiti, A. (2021) Complete remission of follicular lymphoma after SARS-CoV-2 infection: from the "flare phenomenon" to the "abscopal effect". European journal of nuclear medicine and molecular imaging, 48, 2652-2654.

4. Ferguson, M.S., Lemoine, N.R. and Wang, Y. (2012) Systemic delivery of oncolytic viruses: hopes and hurdles. Advances in virology, 2012, 805629.

5. Lundstrom, K. (2019) RNA Viruses as Tools in Gene Therapy and Vaccine Development. Genes, 10.

6. Cai, J. and Yan, G. (2021) The Identification and Development of a Novel Oncolytic Virus: Alphavirus M1. Human gene therapy, 32, 138-149.

7. Anderson, B.D., Nakamura, T., Russell, S.J. and Peng, K.W. (2004) High CD46 receptor density determines preferential killing of tumor cells by oncolytic measles virus. Cancer research, 64, 4919-4926.

8. Mahoney, Douglas J., Lefebvre, C., Allan, K., Brun, J., Sanaei, Cina A., Baird, S., Pearce, N., Grönberg, S., Wilson, B., Prakesh, M. et al. (2011) Virus-Tumor Interactome Screen Reveals ER Stress Response Can Reprogram Resistant Cancers for Oncolytic Virus-Triggered Caspase-2 Cell Death. Cancer Cell, 20, 443-456.

9. Zamarin, D. and Palese, P. (2012) Oncolytic Newcastle disease virus for cancer therapy: old challenges and new directions. Future microbiology, 7, 347-367.

10. Roshal, M., Wood, B.L. and Fromm, J.R. (2011) Flow cytometric detection of the classical hodgkin lymphoma: clinical and research applications. Advances in hematology, 2011, 387034.

11. Nozawa, Y., Wakasa, H. and Abe, M. (1998) Costimulatory molecules (CD80 and CD86) on Reed-Sternberg cells are associated with the proliferation of background T cells in Hodgkin's disease. Pathology international, 48, 10-14.

12. Tzankov, A., Zimpfer, A., Pehrs, A.C., Lugli, A., Went, P., Maurer, R., Pileri, S. and Dirnhofer, S. (2003) Expression of B-cell markers in classical hodgkin lymphoma: a tissue microarray analysis of 330 cases. Modern pathology : an official journal of the United States and Canadian Academy of Pathology, Inc, 16, 1141-1147. 
13. Hofmann, A., Thiesler, T., Gerrits, B., Behnke, S., Sobotzki, N., Omasits, U., Bausch-Fluck, D., Bock, T., Aebersold, R., Moch, H. et al. (2015) Surfaceome of classical Hodgkin and non-Hodgkin lymphoma. Proteomics. Clinical applications, 9, 661-670.

14. Patel, S.S., Weirather, J.L., Lipschitz, M., Lako, A., Chen, P.H., Griffin, G.K., Armand, P., Shipp, M.A. and Rodig, S.J. (2019) The microenvironmental niche in classic Hodgkin lymphoma is enriched for CTLA-4-positive T cells that are PD-1-negative. Blood, 134, 2059-2069.

15. Das, D.K. (2018) Contribution of Immunocytochemistry to the Diagnosis of Usual and Unusual Lymphoma Cases. Journal of cytology, 35, 163-169.

16. van de Donk, N.W. and Dhimolea, E. (2012) Brentuximab vedotin. mAbs, 4, 458-465.

17. Liu, W.R. and Shipp, M.A. (2017) Signaling pathways and immune evasion mechanisms in classical Hodgkin lymphoma. Hematology. American Society of Hematology. Education Program, 2017, 310-316.

18. Nath, K. and Gandhi, M.K. (2021) Targeted Treatment of Follicular Lymphoma. Journal of personalized medicine, 11.

19. Fessas, P., Lee, H., Ikemizu, S. and Janowitz, T. (2017) A molecular and preclinical comparison of the PD-1-targeted T-cell checkpoint inhibitors nivolumab and pembrolizumab. Seminars in oncology, 44, 136-140.

20. Voorhees, T.J. and Beaven, A.W. (2020) Therapeutic Updates for Relapsed and Refractory Classical Hodgkin Lymphoma. Cancers, 12.

21. Vockerodt, M., Cader, F.Z., Shannon-Lowe, C. and Murray, P. (2014) Epstein-Barr virus and the origin of Hodgkin lymphoma. Chinese journal of cancer, 33, 591-597.

22. Murray, P.G. and Young, L.S. (2019) An etiological role for the Epstein-Barr virus in the pathogenesis of classical Hodgkin lymphoma. Blood, 134, 591-596.

23. Mackrides, N., Campuzano-Zuluaga, G., Maque-Acosta, Y., Moul, A., Hijazi, N., Ikpatt, F.O., Levy, R., Verdun, R.E., Kunkalla, K., Natkunam, Y. et al. (2017) Epstein-Barr virus-positive follicular lymphoma. Modern pathology : an official journal of the United States and Canadian Academy of Pathology, Inc, 30, 519-529.

24. Kieser, A. (2007) Signal transduction by the Epstein-Barr virus oncogene latent membrane protein 1 (LMP1). Signal Transduction, 7, 20-33.

25. Yang, J. and Zhang, Y. (2015) I-TASSER server: new development for protein structure and function predictions. Nucleic acids research, 43, W174-181.

26. Källberg, M., Wang, H., Wang, S., Peng, J., Wang, Z., Lu, H. and Xu, J. (2012) Template-based protein structure modeling using the RaptorX web server. Nature protocols, 7, 1511-1522.

27. Ko, J., Park, H., Heo, L. and Seok, C. (2012) GalaxyWEB server for protein structure prediction and refinement. Nucleic acids research, 40, W294-297.

28. Pierce, B.G., Wiehe, K., Hwang, H., Kim, B.H., Vreven, T. and Weng, Z. (2014) ZDOCK server: interactive docking prediction of protein-protein complexes and symmetric multimers. Bioinformatics (Oxford, England), 30, 1771-1773.

29. Yan, Y., Zhang, D., Zhou, P., Li, B. and Huang, S.Y. (2017) HDOCK: a web server for proteinprotein and protein-DNA/RNA docking based on a hybrid strategy. Nucleic acids research, 45, W365-w373.

30. Laskowski, R.A. and Swindells, M.B. (2011) LigPlot+: multiple ligand-protein interaction diagrams for drug discovery. Journal of chemical information and modeling, 51, 2778-2786.

31. Pettersen, E.F., Goddard, T.D., Huang, C.C., Couch, G.S., Greenblatt, D.M., Meng, E.C. and Ferrin, T.E. (2004) UCSF Chimera--a visualization system for exploratory research and analysis. Journal of computational chemistry, 25, 1605-1612.

32. Hargreaves, P.G. and Al-Shamkhani, A. (2002) Soluble CD30 binds to CD153 with high affinity and blocks transmembrane signaling by CD30. European journal of immunology, 32, 163-173.

33. Dong, L., Hülsmeyer, M., Dürkop, H., Hansen, H.P., Schneider-Mergener, J., Ziegler, A. and Uchanska-Ziegler, B. (2003) Human CD30: structural implications from epitope mapping and modeling studies. Journal of molecular recognition : JMR, 16, 28-36.

34. Huang, Y., Yang, C., Xu, X.F., Xu, W. and Liu, S.W. (2020) Structural and functional properties of SARS-CoV-2 spike protein: potential antivirus drug development for COVID-19. Acta pharmacologica Sinica, 41, 1141-1149.

35. Shang, J., Ye, G., Shi, K., Wan, Y., Luo, C., Aihara, H., Geng, Q., Auerbach, A. and Li, F. (2020) Structural basis of receptor recognition by SARS-CoV-2. Nature, 581, 221-224.

36. Yan, R., Zhang, Y., Li, Y., Xia, L., Guo, Y. and Zhou, Q. (2020) Structural basis for the recognition of SARS-CoV-2 by full-length human ACE2. Science (New York, N.Y.), 367, 1444-1448.

37. Barh, D., Tiwari, S., Silva Andrade, B., Giovanetti, M., Almeida Costa, E., Kumavath, R., Ghosh, P., Góes-Neto, A., Carlos Junior Alcantara, L. and Azevedo, V. (2020) Potential chimeric peptides to block the SARS-CoV-2 spike receptor-binding domain. F1000Research, 9, 576.

38. Gordon, D.E., Jang, G.M., Bouhaddou, M., Xu, J., Obernier, K., White, K.M., O'Meara, M.J., Rezelj, V.V., Guo, J.Z., Swaney, D.L. et al. (2020) A SARS-CoV-2 protein interaction map reveals targets for drug repurposing. Nature, 583, 459-468. 
39. Chen, Z., Wang, C., Feng, X., Nie, L., Tang, M., Zhang, H., Xiong, Y., Swisher, S.K., Srivastava, $M$. and Chen, J. (2021) Comprehensive analysis of the host-virus interactome of SARS-CoV-2. bioRxiv, 2020.2012.2031.424961.

40. Oakley, B.R., Paolillo, V. and Zheng, Y. (2015) Y-Tubulin complexes in microtubule nucleation and beyond. Molecular biology of the cell, 26, 2957-2962.

41. Wieczorek, M., Urnavicius, L., Ti, S.C., Molloy, K.R., Chait, B.T. and Kapoor, T.M. (2020) Asymmetric Molecular Architecture of the Human y-Tubulin Ring Complex. Cell, 180, 165$175 . \mathrm{e} 116$.

42. Lee, J.Y., Lee, H.T., Shin, W., Chae, J., Choi, J., Kim, S.H., Lim, H., Won Heo, T., Park, K.Y., Lee, Y.J. et al. (2016) Structural basis of checkpoint blockade by monoclonal antibodies in cancer immunotherapy. Nature communications, 7, 13354.

43. Tan, S., Zhang, H., Chai, Y., Song, H., Tong, Z., Wang, Q., Qi, J., Wong, G., Zhu, X., Liu, W.J. et al. (2017) An unexpected N-terminal loop in PD-1 dominates binding by nivolumab. Nature communications, 8, 14369.

44. Barh, D., Tiwari, S., Andrade, B.S., Weener, M.E., Góes-Neto, A., Azevedo, V., Ghosh, P., Blum, K. and Ganguly, N.K. (2021) A novel multi-omics-based highly accurate prediction of symptoms, comorbid conditions, and possible long-term complications of COVID-19. Molecular omics, 17, 317-337.

45. Barh, D., Aljabali, A.A., Tambuwala, M.M., Tiwari, S., Serrano-Aroca, Á., Alzahrani, K.J., Silva Andrade, B., Azevedo, V., Ganguly, N.K. and Lundstrom, K. (2021) Predicting COVID-19Comorbidity Pathway Crosstalk-Based Targets and Drugs: Towards Personalized COVID-19 Management. Biomedicines, 9, 556.

46. Buckner, T.W., Dunphy, C., Fedoriw, Y.D., van Deventer, H.W., Foster, M.C., Richards, K.L. and Park, S.I. (2012) Complete spontaneous remission of diffuse large B-cell lymphoma of the maxillary sinus after concurrent infections. Clinical lymphoma, myeloma \& leukemia, 12, 455-458.

47. Rudolphi-Solero, T., Rashki, M., Fernández-Fernández, J., Rivas-Navas, D., Ramos-Font, C. and Rodríguez-Fernández, A. (2021) El virus SARS-COV-2 desencadena una respuesta inmunitaria antitumoral en un paciente con linfoma. Revista espanola de medicina nuclear e imagen molecular.

48. O'Kelly, B., McGettrick, P., Angelov, D., Fay, M., McGinty, T., Cotter, A.G., Sheehan, G. and Lambert, J.S. (2020) Outcome of a patient with refractory Hodgkin lymphoma on pembrolizumab, infected with SARS-CoV-2. British journal of haematology, 190, e1-e3.

49. Kanna, A., Agrawal, S., Jayant, K., Kumar Pala, V., Altujjar, M., Hadid, T. and Khurram, M. (2015) B Cell Lymphoma, Unclassifiable, Transformed from Follicular Lymphoma: A Rare Presentation with Review of the Literature. Case reports in hematology, 2015, 651764.

50. Dong, H.Y., Shahsafaei, A. and Dorfman, D.M. (2002) CD148 and CD27 are expressed in B cell lymphomas derived from both memory and naïve B cells. Leukemia \& lymphoma, 43, 1855-1858.

51. Galanina, N., Kline, J. and Bishop, M.R. (2017) Emerging role of checkpoint blockade therapy in lymphoma. Therapeutic advances in hematology, 8, 81-90.

52. Chuang, S.S. (2017) Infrequent expression of CD15 by classical Hodgkin's lymphomas in Taiwan. Journal of clinical pathology, 70, 183-184.

53. Jones, R.J., Gocke, C.D., Kasamon, Y.L., Miller, C.B., Perkins, B., Barber, J.P., Vala, M.S., Gerber, J.M., Gellert, L.L., Siedner, M. et al. (2009) Circulating clonotypic B cells in classic Hodgkin lymphoma. Blood, 113, 5920-5926.

54. Bosler, D.S., Douglas-Nikitin, V.K., Harris, V.N. and Smith, M.D. (2008) Detection of T-regulatory cells has a potential role in the diagnosis of classical Hodgkin lymphoma. Cytometry. Part B, Clinical cytometry, 74, 227-235.

55. Chen, Z., Wang, C., Feng, X., Nie, L., Tang, M., Zhang, H., Xiong, Y., Swisher, S.K., Srivastava, M. and Chen, J. (2021) Comprehensive analysis of the host-virus interactome of SARS-CoV-2. bioRxiv, 2020-2012.

56. Gioti, K., Kottaridi, C., Voyiatzaki, C., Chaniotis, D., Rampias, T. and Beloukas, A. (2021) Animal Coronaviruses Induced Apoptosis. Life (Basel, Switzerland), 11.

57. Chu, H., Shuai, H., Hou, Y., Zhang, X., Wen, L., Huang, X., Hu, B., Yang, D., Wang, Y., Yoon, C. et al. (2021) Targeting highly pathogenic coronavirus-induced apoptosis reduces viral pathogenesis and disease severity. Science advances, 7.

58. Kopecky-Bromberg, S.A., Martinez-Sobrido, L. and Palese, P. (2006) 7a protein of severe acute respiratory syndrome coronavirus inhibits cellular protein synthesis and activates p38 mitogenactivated protein kinase. Journal of virology, 80, 785-793.

59. Chow, K.Y., Yeung, Y.S., Hon, C.C., Zeng, F., Law, K.M. and Leung, F.C. (2008) SARS coronavirus and apoptosis. Hong Kong medical journal = Xianggang yi xue za zhi, 14 Suppl 4, 813.

60. Zhao, G., Shi, S.Q., Yang, Y. and Peng, J.P. (2006) M and N proteins of SARS coronavirus induce apoptosis in HPF cells. Cell biology and toxicology, 22, 313-322. 
61. Ren, Y., Shu, T., Wu, D., Mu, J., Wang, C., Huang, M., Han, Y., Zhang, X.Y., Zhou, W., Quu, Y. et al. (2020) The ORF3a protein of SARS-CoV-2 induces apoptosis in cells. Cellular \& molecular immunology, 17, 881-883.

62. Barh, D., Tiwari, S., Weener, M.E., Azevedo, V., Góes-Neto, A., Gromiha, M.M. and Ghosh, P. (2020) Multi-omics-based identification of SARS-CoV-2 infection biology and candidate drugs against COVID-19. Computers in biology and medicine, 126, 104051.

63. García-Martínez, F.J., Moreno-Artero, E. and Jahnke, S. (2020) SARS-CoV-2 and EBV coinfection. Medicina clinica (English ed.), 155, 319-320.

64. Paolucci, S., Cassaniti, I., Novazzi, F., Fiorina, L., Piralla, A., Comolli, G., Bruno, R., Maserati, R., Gulminetti, R., Novati, S. et al. (2021) EBV DNA increase in COVID-19 patients with impaired lymphocyte subpopulation count. International journal of infectious diseases : IJID : official publication of the International Society for Infectious Diseases, 104, 315-319.

65. Chen, T., Song, J., Liu, H., Zheng, H. and Chen, C. (2021) Positive Epstein-Barr virus detection in coronavirus disease 2019 (COVID-19) patients. Scientific reports, 11, 10902.

66. Gold, J.E., Okyay, R.A., Licht, W.E. and Hurley, D.J. (2021) Investigation of Long COVID Prevalence and Its Relationship to Epstein-Barr Virus Reactivation. Pathogens (Basel, Switzerland), 10.

67. Roncati, L., Lusenti, B., Nasillo, V. and Manenti, A. (2020) Fatal SARS-CoV-2 coinfection in course of EBV-associated lymphoproliferative disease. Annals of hematology, 99, 1945-1946. 